\title{
Preparation for endoscopic examinations in patients with diabetes and hypoglycaemia
}

\author{
Konrad Lewandowski ${ }^{1}$, Grażyna Rydzewska ${ }^{1,2}$, Tomasz K. Ledwoń ${ }^{3}$ \\ ${ }^{1}$ Clinical Department of Internal Medicine and Gastroenterology with Inflammatory Bowel Disease Unit, Central Clinical \\ Hospital of the Ministry of the Inferior and Administration, Warsaw, Poland \\ ${ }^{2}$ Collegium Medicum, Jan Kochanowski University, Kielce, Poland \\ ${ }^{3}$ BioGliko Sp. z o.o., Warsaw, Poland
}

Gastroenterology Rev 2021; 16 (4): 297-305

DOI: https://doi.org/10.5114/pg.2021.111763

Key words: diabetes, metabolic disorders, preparation for colonoscopy.

Address for correspondence: Konrad Lewandowski, Clinical Department of Internal Medicine and Gastroenterology with Inflammatory Bowel Disease Unit, Central Clinical Hospital of the Ministry of the Inferior and Administration, Warsaw, 137 Wołska St, 02-507 Warsaw, e-mail: dr.k.lewandowski@icloud.com

\begin{abstract}
Endoscopy is a procedure used for diagnostic and therapeutic purposes, which requires proper preparation. Patients with diabetes mellitus are particularly predisposed to inadequate cleansing for endoscopy due to slowing bowel movements, dietary preparation restrictions, glucose reduction, and the resulting symptoms of hypoglycaemia. So far, no comprehensive guidelines of preparation for endoscopic examinations for patients with these metabolic disorders have been developed. Considering the number of patients who may be subjected to colonoscopy and the possible effects of suboptimal preparation for the examination, the lack of such recommendations seems to be a significant gap in the healthcare system. The article reviews the literature on the above topic.
\end{abstract}

\section{Introduction}

Correct preparation of the bowel is a prerequisite for proper endoscopic examination of the lower gastrointestinal tract and for obtaining diagnostic results. The most important and necessary element of patient preparation for the examination is to stop consuming solid and liquid foods within a specified time before it and - in the case of lower digestive system endoscopy - to use oral laxatives [1]. Improper bowel cleansing does not allow for an accurate assessment of the mucosa, significantly reducing the diagnostic value of the examination, increasing the risk of complications, and missing neoplastic changes. The total cost incurred to conduct the procedure is increased due to the need to repeat it or extend its duration $[2,3]$.

It is estimated that approximately $25 \%$ of cases are characterized by improper preparation of the bowel for the examination [4]. This may result in the following: - reduced visibility of polyps and, consequently, lower adenoma detection rate (ADR) [5];
- longer time necessary to complete the procedure;

- lower caecal intubation rate (CIR);

- the need to implement more frequent examination repetitions [4];

- missed small or flat lesions;

- a significant obstacle in the access of the colonoscope to individual segments of the bowel;

- increased likelihood of complications;

- more sedatives and analgesics being required [5].

Some patients who fail colonoscopy due to insufficient bowel cleansing are indicated for a repeat procedure $[2,6]$. Repeat colonoscopy has consequences for the health care system - increasing costs and reducing access to the procedure, as well as for the patient decreasing the quality of life due to the need to repeat preparation, which is considered unpleasant, and the examination itself, which is burdensome due to its invasiveness [2]. Improper bowel cleansing extends the duration of the entire procedure and is associated with an increased risk of adverse events [6, 7]. Moreover, the necessity to repeat the examination reduces its avail- 
ability, thus extending the waiting time for other patients [2]. An analysis carried out in the United States showed that colonoscopies are not cost-effective if the percentage of inadequately prepared patients exceeds $13 \%$ [6]. It was shown that at least one adenoma was detected in $33.8 \%$ of colonoscopies repeated due to inadequate bowel preparation [6]. A study conducted in Poland on 165,000 patients who had colonoscopy under the Polish Colon Cancer Screening Program proved that only high-quality examination provided a reduction of the risk of colon cancer $[8,9]$.

Special recommendations apply to groups of patients at high risk of improper preparation for the examination, i.e. people with certain diseases or using certain groups of medicines in the long-term. It was shown that some diseases (constipation, diabetes, hypertension, cirrhosis, stroke, and dementia) and medications (opioid analgesics and tricyclic antidepressants) may constitute risk factors for colonoscopy failure. In these patients, additional measures should be taken to increase the safety as well as the effectiveness of bowel cleansing $[1,10]$.

In most studies, diabetes is mentioned as one of the main reasons of poorer bowel preparation, as well as a potential reason for a repeat colonoscopy [11]. During the preparation for endoscopy the risk of hypoglycaemia occurrence is high in both types of diabetes. Another large group of patients who require an adapted bowel preparation procedure for endoscopic examinations are patients with reactive hypoglycaemia. Likewise, patients suffering from hypothyroidism, hypopituitarism, or adrenal insufficiency are also among those at risk of developing hypoglycaemia.

The above-mentioned groups of patients are at risk of hypoglycaemia as well as fluid and electrolyte imbalance, acute renal failure, lactic acidosis, and ketoacidosis. Reductions in the glucose level and the resulting symptoms can lead to deviations from the correct preparation procedure or, in extreme cases, to its discontinuation. Therefore, in this group of patients it is required to modify not only the appropriate recommendations regarding the use of antihyperglycaemic agents, but also the dietary recommendations in order to enable effective, safe, and individualized preparation for the examination [10-12].

The aim of the study was to review the methods of preparing patients for endoscopic examinations, as well as the reasons for inadequate preparation in patients with metabolic disorders, primarily diabetes and hypoglycaemia, including reactive hypoglycaemia. An attempt was also made to characterize the profile of a patient who, due to metabolic disorders, may encounter difficulties in proper preparation for endoscop- ic examinations and other procedures requiring bowel cleansing.

\section{Epidemiology and the scale of the problem}

Based on the billing data of medical consultations and reimbursed drugs, the number of patients suffering from diabetes in Poland in 2013 was determined at 2.17 million, which constituted $5.6 \%$ of the total number of inhabitants of Poland. According to the results of the NATPOL 2011 study, it has been estimated that $26 \%$ of people with diabetes are unaware of their disease. Therefore, the total number of people suffering from diabetes in 2013 could be as high as 2.73 million $[13,14]$. In turn, the data of the International Diabetes Federation (IDF) indicate that in the case of the Polish population, approximately 2.48 million people aged 18 to 99 years suffer from diabetes, which constitutes $7.8 \%$ of the entire population at this age in Poland. Considering that this document also covers the paediatric population, the number of patients with diabetes in general may be much larger [15]. According to the National Health Fund, in 2018 there were 2.9 million adults with diabetes in Poland, so as many as one in every eleven adults suffered from the disease. These data refer to diagnosed people, while some people have pre-diabetes (glucose concentration in the range of $100-125 \mathrm{mg} / \mathrm{dl}$ in a fasting blood test) or are not aware of their disease $[8,9]$.

Due to the lack of data on the incidence of hypoglycaemia in Poland, literature reports were used. They indicate that a significant proportion of patients who experience hypoglycaemia do not have typical clinical symptoms. Three studies conducted in the UK, Canada, and Denmark found that only $23 \%, 47 \%$, and $0 \%$ of patients, respectively, had a glucose level $\leq 3.3 \mathrm{mmol} / \mathrm{l}$ despite symptoms of hypoglycaemia. In another British study, the incidence of symptomatic hypoglycaemia self-reported by patients (occurring 4 times a month) was observed in $37.9 \%$ of women aged $17-50$ years [16]. Another British study (conducted on randomly selected women from Nottinghamshire) indicated that only $0.5 \%$ of women reporting symptoms of hypoglycaemia had sought medical advice. However, over a third of participants of this study reported symptoms of hypoglycaemia, and in $18 \%$ of them these symptoms occurred more frequently than once a week [17]. The incidence of hypoglycaemia in hospitalized patients without diagnosed diabetes was also investigated. Out of almost 38,000 patients admitted to hospital in the UK, hypoglycaemia occurred in 36 out of 10,000 inpatient admissions. The following most common causes of hypoglycaemia were pointed out: taking medication, 
alcohol consumption, and co-morbidities (e.g. liver, kidney, or heart failure, sepsis, or loss of consciousness); in most cases more than one cause of hypoglycaemia was present. Hypoglycaemia without any specific cause occurred in 7 patients only [18]. A similar incidence of non-diabetic hypoglycaemia among inpatients was observed in another study conducted in Japan [19].

Data on the number of endoscopic examinations conducted in Poland are limited. The results of a study published in 2009 concerning the performance of endoscopic procedures in Poland indicated that, on the basis of 138 questionnaires sent out to endoscopy laboratories and returned, the average number of gastroscopies performed per week was 25.7 ( \pm 14.79$)$, and colonoscopies $-17.4( \pm 20.04)$. Only some of these examinations (gastroscopy - about 7\%, colonoscopy - 18.4\%) were therapeutic procedures: polypectomy, SEMS (Self-expanding metal stents), or bleeding control [20]. In 2000, the number of screening colonoscopies performed in Poland was 14,000 annually; currently the number of colonoscopies performed annually is 122,000 . Statistics on the number of examinations performed in the years 2000-2019 as part of the CoIon Cancer Screening Program indicate that 102,000 colonoscopies were performed in 2018, and 67,000 in 2019. The total number of colonoscopies performed annually is estimated at 500,000.

The demand for colonoscopy in diabetic patients is higher than in the general population due to the frequent occurrence of gastrointestinal symptoms and an increased risk of colorectal cancer $[11,21]$. Considering the above reports, the problem of patients with carbohydrate metabolism disorders is significant. Additionally, the fact that the data are not accurate and are certainly underestimated is also challenging.

\section{Difficulties and threats concerning patients with disorders of carbohydrate metabolism}

The guidelines for standard preparation for endoscopy emphasize the importance of educating patients about appropriate preparation for examination, regardless of whether the examination concerns the upper or lower gastrointestinal tract. In the case of oesophagogastroduodenoscopy, fasting is recommended for 6$8 \mathrm{~h}$ prior to the examination. As regards preparing for colonoscopy, it is recommended that a non-residual or liquid diet be used and appropriate bowel cleansing agents (mainly polyethylene glycol) be taken as part of the split-dose method, a method based on dividing the full dose into portions used the day before the colonoscopy and on the day of its conducting, or - if a colonoscopy is performed in the afternoon - requir- ing use of the full dose of the bowel cleansing agent of the same day.

During the preparation for colonoscopy, diabetic patients are exposed to many risks related to the need to change their diet, change the regimen of using antidiabetic drugs, and complications related to diabetes. These include hypoglycaemia, fluid and electrolyte imbalance, acute renal failure, lactic acidosis, and ketoacidosis. The occurrence of any of the above complications often results in extension of the hospitalization time, increases the amount of stress, and adversely affects the well-being of patients $[3,11]$. So far, no comprehensive recommendations for endoscopic examination of patients with metabolic disorders have been developed [3]. Given the number of people who will potentially undergo colonoscopy and the possible effects of suboptimal preparation for the examination, the lack of such recommendations seems to be a significant gap in the healthcare system as regards the patients with indication for endoscopic examinations. This is reflected in the fact that hospitals and centres that conduct endoscopic examinations often independently develop such recommendations, which, however, do not constitute clinical guidelines and are usually very general, allowing a patient to interpret them freely.

\section{Effectiveness of colonoscopy preparation procedures in the diabetic patient population}

A prospective, blind study compared the effectiveness of bowel cleansing before colonoscopy in a population of healthy adults to those with diabetes treated with insulin or oral medications. The procedure of preparation for the examination included the use of a liquid diet (clear liquids), taking 6 I of GoLYTELY and 2 laxative tablets (Dulcolax) the day before the planned examination. The primary outcome measure in the study was the quality of bowel preparation score, rated on a 14-point scale based on the surface area of the mucosa that could be estimated, and the presence and consistency of residual stool. In the control group (healthy people, $n=54$ ), $97 \%$ of the participants were rated good or better, while in the study group (diabetic patients, $n=45$ ), such a rate was obtained by $62 \%(p<0.001)$. There was not a single case requiring repeat colonoscopy in the control group, whereas $9 \%$ of diabetic patients were referred for re-examination due to the insufficient quality of bowel cleansing $(p<0.01)$ [22].

Another prospective study evaluated the effect of autonomous neuropathy on bowel preparation in type 2 diabetes mellitus (DM) patients. In order to prepare for the colonoscopy, both the study group (DM, $n=45$ ) 
and the control group (non-diabetic, $n=48$ ) were assigned to oral sodium phosphate solution $(2 \times 45 \mathrm{ml}$ with a 12-hour interval). The quality of bowel cleansing was rated using the Aronchick scale and ranged from inadequate (re-preparation required) to excellent. With regard to the bowel preparation quality, optimal bowel cleansing was achieved in $73.3 \%$ of diabetic and $93.8 \%$ of non-diabetic patients, and the difference was statistically significant $(p<0.05)$. Optimal bowel cleansing was achieved in $42.8 \%$ of diabetes patients with neuropathy and in $87.1 \%$ of diabetes patients without neuropathy, and the difference was statistically significant. In the neuropathic group, the time to reach the caecum and the overall examination duration was longer than in the non-neuropathic group. This may indicate that the presence of neuropathy in diabetic patients is a predictor of poor preparation for colonoscopy [23].

In 2010, Ozturk et al. compared the effectiveness of bowel cleansing with the use of sodium phosphate in a group of healthy subjects and patients with diabetes. The Aronchick scale was used to rate the quality of preparation for the colonoscopy procedure. The bowel was rated as properly cleaned in $70 \%$ of the study group (diabetic patients, $n=50$ ) and in $94 \%$ of the control group (healthy individuals, $n=50)(p=0.002)$. An inadequate rate of bowel cleansing was observed in $6 \%$ of the control group and in $30 \%$ of the diabetic subjects $(p<0.05)[23]$.

In a study published in 2017 Hildsen et al. examined the impact of scheduling patients for colonoscopy (7:30-9:30 a.m. vs. after 9:30 a.m.) on the quality of bowel preparation in groups of patients with and without diabetes. In the centre that conducted the colonoscopies, in 2013 most diabetic patients were scheduled between 7:30 and 9:30 a.m., while in the next year the scheduling was changed to 9:30 (it is worth noting that the centre carried out a 2-stage preparation for the examination, where the second stage was to be carried out 4-6 $\mathrm{h}$ before the examination). In the case of colonoscopy performed between 7:30 a.m. and 9:30 a.m., inadequate quality of bowel cleansing was observed in $7.7 \%$ of diabetic patients, and after switching to later hours, the percentage of inadequately prepared diabetic patients decreased to $3.2 \%$, which indicates that in the case of the split-dose preparation of patients with diabetes, scheduling colonoscopy at later hours has a positive impact on the quality of bowel preparation. Such a relationship was not observed in the population of patients without diabetes, where the percentage of patients inadequately prepared for the examination was $1.9 \%$ and $1.7 \%$ in 2013 and 2014 , respectively. There is another relationship that can be observed on the basis of the above study: even in a centre with precisely defined procedures of preparation for colonoscopy the percentage of patients with diabetes prepared improperly was higher than in those without diabetes [24].

In a prospective study published in $2017 \mathrm{Kim}$ et al. reported that bowel cleansing for colonoscopy with use of a polyethylene glycol (PEG) split regimen divided between 2 days is ineffective in adult patients with diabetes. The primary outcome, i.e. the bowel cleansing efficacy, was assessed using the Ottawa scale. Secondary study outcomes included the degree of preparation completion, patient tolerability, adverse events, procedure time, and polyp/adenoma detection. In $70 \%$ of the control group (healthy subjects, $n=55$ ), the quality of bowel preparation was rated as adequate (Ottawa score from 0 to 6 ). The same score was observed in $40 \%$ of the subjects in the study group (diabetic patients, $n=50$ ), $(p=0.003)$. The efficacy of the quality of cleansing of particular parts of the intestine showed statistically significantly better preparation for colonoscopy among the subjects from the control group (excluding the scores of the mid colon, where the difference between the groups was not significant). There was a statistically significant difference in the duration of the examination and in the caecal intubation time; both times were longer in the group of diabetic patients (duration of colonoscopy: $22.1( \pm 7.6)$ min vs. $18.1( \pm 8.5) \min , p=0.015$, caecal intubation time: $6.4( \pm 3.6) \mathrm{min}$ vs. $4.5( \pm 2.4) \mathrm{min}, p=0.002$, diabetic patients vs. controls). A higher polyp detection rate was also observed in the study group compared to the control group, despite the comparable number of polyps per patient in both groups ( $82 \%$ vs. $62 \%, p=$ 0.019 and $2.3( \pm 2.4)$ vs. $2.1( \pm 3.7), p=0.835$, diabetic patients vs. controls). Analysis of the other secondary outcomes showed no statistically significant differences between the group of diabetic patients and healthy subjects [25].

A study by Ozturk et al. (2010) examined the influence of some risk factors in diabetic patients on the quality of bowel preparation for colonoscopy; the subjects of the analysis were as follows: age, disease duration, presence of late diabetes complications, and fasting glucose and glycated glucose $\left(\mathrm{HbA}_{1 \mathrm{c}}\right)$ levels. Advanced age, long disease duration, and the presence of late diabetic complications significantly increase the risk of improper bowel cleansing. A similar relationship was observed for diagnostic parameters (fasting glucose, $\mathrm{HbA}_{1 \mathrm{c}}$ level), which could suggest improperly conducted hypoglycaemic treatment. Proper bowel cleansing was not related to gender or body mass index (BMI) [23]. 
The influence of the hypoglycaemic treatment on the quality of preparation for colonoscopy was also analysed. In a retrospective study performed in 2017 by Madhoun et al., the effects of diabetes and narcotics on the bowel preparation were analysed. The study participants were divided into 4 groups: no diabetes, no narcotics $(n=1512)$; diabetes only $(n=600)$; non-diabetics using narcotics $(n=516)$; diabetics on narcotics $(n=213)$. All patients received a polyethylene glycol-based bowel preparation (from 2014 as a split dose on the day of the procedure and the day preceding it, previously in 1 dose on the day before the procedure). Quality of bowel preparation was scored using the Boston Scale (BBPS). The result of "excellent" bowel preparation for colonoscopy was obtained by $54 \%$ of the control group (healthy participants of the study), $38 \%$ of diabetics and those using narcotics, $44 \%$ of those without diabetes but using narcotics, and $48 \%$ of diabetic patients $(p<0.0001)$. The study showed that both diabetes and the use of narcotics had a negative effect on the bowel cleansing rate, and the coexistence of both factors intensified this effect. The authors of the study suggested that patients with more than 1 risk factor for poorer bowel cleansing should receive special care at the stage of preparation for colonoscopy. The authors also pointed to the need to adapt the preparatory procedures to the individual needs of a person referred for this examination [26].

The Hochberg article from 2019 indicates that certain groups of medications, e.g. antihypertensive medications often used by patients with diabetes, may affect intestinal motility and cause constipation [11]. Likewise, it may be the case of the GLP-1 receptor agonists, which slow the intestinal motility and have the potential to increase the risk of poor bowel preparation.

The 2018 Mahmood systematic review and meta-analysis of available data from 24 studies on risk factors for colonoscopy failure indicated that there is a relationship between colonoscopy improper bowel preparation and the following:

- age $(O R=-1.20)$;

- male sex $(O R=0.85)$;

- inpatient status $(O R=0.57)$;

- diabetes mellitus $(\mathrm{OR}=0.58)$;

- hypertension $(\mathrm{OR}=0.58)$;

- cirrhosis $(O R=0.49)$;

- narcotic use $(O R=0.59)$;

- constipation $(\mathrm{OR}=0.61)$;

- stroke $(O R=0.51)$;

- tricyclic antidepressants (TCA) $(O R=0.51)$ [27].

In this article, the cumulative frequency of inadequate preparation for colonoscopy in diabetic patients was $22.8 \%$ (827/3620), while the cumulative frequen- cy of adequate preparation was $13.4 \%$ (1303/9082). It was also shown that the presence of diabetes is a predictor of poor preparation for colonoscopy in Western countries (as opposed to Asian countries), regardless of the preparation scheme (i.e. single vs. split dose).

The reasons for inadequate bowel preparation can also be found in the lack of sufficient education of patients in this area. Therefore, it is reasonable to use tools informing patients about the need to use an appropriate diet at a specific time before the procedure. Such tools include the following: instructional leaflets, visual aids, information brochures, video materials, interviews with medical staff (doctor, nurse), and mobile applications.

\section{Methods of mitigating the risk of hypoglycaemia and metabolic disorders}

A randomized, single-blind study conducted in an adult population with type II diabetes evaluated a specially designed protocol of preparation for endoscopic examination modified for patients with diabetes, which included dietary recommendations and appropriate selection of glucose lowering agents to prevent hypoglycaemia, as well as educational intervention. The primary outcome measure in the study was inadequate bowel preparation according to the Boston scale, in which a score of less than 2 indicated inadequate bowel preparation. It was shown that in the group of patients using the modified diabetes protocol $(n=76)$ the percentage of patients with improperly prepared bowel was statistically significantly lower than in the group of patients using the standard protocol $(n=74)$, and it amounted to $7 \%$ and $20 \%$, respectively ( $p=0.014, \mathrm{RR}=$ $3.1,95 \% \mathrm{Cl}: 1.2-8)$. In the group using the modified protocol there was a slight trend towards better tolerance of the procedure $(p=0.075)$. Only 4 episodes of symptomatic hypoglycaemia were reported: 3 in the standard protocol group and 1 in the modified protocol group $(p=0.3)$. In the multivariate analysis, independent predictors of inadequate bowel preparation in diabetics were the use of the standard protocol $(\mathrm{OR}=3.5,95 \% \mathrm{Cl}$ : 1.2-10.4) and the patient's performance status on the ECOG scale of $>1(\mathrm{OR}=3.8,95 \% \mathrm{Cl}: 1.4-10.2)$ [12].

Similar results demonstrating the benefits of use of modified endoscopy preparation protocols in diabetic patients were observed in a 2011 randomized, single-blind study designed by Hayes et al. In this study, the experimental method of preparation for colonoscopy (which differs, inter alia, in earlier preparation time for the examination and earlier use of a bowel cleansing agent) was compared to the standard protocol. In over 2 years of study it was observed that in the study 
group ( $n=106)$ "good" preparation for the examination was observed in $69.7 \%$ of patients, while in the control group $(n=92)$ it was seen in $53.5 \%$ of patients $(p=0.02)$. However, the study did not evaluate the impact of the applied protocols on the occurrence of hypoglycaemia episodes [28].

In 2010 Grigg et al. conducted a randomized, single-blind study in a group of diabetic patients aged $\geq 50$ years, that compared use of polyethylene glycol (PEG) in combination with lubiprostone to PEG alone. The primary measured endpoint of the study was the quality of colon cleansing preparation rated by the endoscopic practitioner on a 5-point Likert scale. It was shown that "very good" or "excellent" quality of bowel cleansing was observed in $8(47.1 \%)$ of the 17 patients in the group using lubiprostone additionally, compared with $6(25.0 \%)$ of the 24 control patients. The difference between the groups was not statistically significant ( $p=0.14$ ), which can probably be attributed to the small size of the groups. The mean score on the modified Ottawa scale in the study group was 2.47 , and in the control group it was $3.00(p=0.09)$. No serious adverse events were reported in the study. The impact of the applied protocols on the occurrence of hypoglycaemic episodes was not assessed [29].

A randomized study published in 2018 by Madhoun et al. in which they evaluated whether adding bisacodyl to the bowel cleansing procedure based on the split-dose protocol would improve the quality of bowel preparation in patients with diabetes. In this study, patients were assigned to groups: the group using a 4-L polyethylene glycol solution with electrolytes as part of the standard preparation protocol (taking the solution during 2-4 h on the day before the procedure, before 6:00 p.m.; $n=63$ ), the group using the split-dose protocol (taking half of the solution at 6:00 p.m. on the day preceding the procedure, and the remaining half in the early morning, at least $3 \mathrm{~h}$ before the scheduled time of the colonoscopy; $n=60$ ), and the group using the split-dose protocol preceded by administration of $10 \mathrm{mg}$ bisacodyl orally (taken at 12.00 p.m. the day before colonoscopy; $n=63$ ). All patients were placed on a liquid diet during the 2 days prior to the colonoscopy. The endoscopists were blinded to the preparation regimen used prior to the examination. The primary outcome in the study was the quality of bowel preparation rated with use of the BBPS scale or subjective rating of the endoscopist. It was shown that most patients achieved adequate bowel preparation (BBPS total score $\geq 6$ points and $\geq 2$ points for all bowel segments): $66.7 \%$ of patients using the standard protocol, $83.3 \%$ of patients who underwent split-method dose protocol, and $74.6 \%$ of patients us- ing additionally bisacodyl. The mean (SD) BBPS score was $6.7( \pm 1.5)$ points in the group assigned to the standard bowel cleansing protocol compared with 7.3 $( \pm 1.6)$ points in the split-dose group, and $7.1( \pm 1.4)$ in the group using additionally bisacodyl. Analysis of the results for each segment of the colon showed that a greater percentage of patients in the group using the standard protocol $(14 / 56,25 \%)$ obtained a bowel right side BBPS score $<2$ points compared to patients using the split-dose method $(5 / 58,10.3 \%)$ and additionally bisacodyl (9/60, 15\%). The difference was close to statistical significance $(p=0.02)$. Most participants in all the procedure arms completed more than a half of their assigned bowel preparation. Approximately twothirds of the study participants felt that preparation for the examination was either easy or neutral. The incidence of procedure-related adverse events was similar between the groups. No serious adverse events were observed. The study did not provide information on the occurrence of hypoglycaemic episodes [26].

In 2017, in a retrospective medical record analysis, Sharma et al. investigated the impact of GLP-1 receptor agonists on the quality of bowel cleansing prior to colonoscopy. The comparison of the group of patients with type II diabetes over 45 years of age taking a GLP-1 agonist $(n=126)$ and those who were not taking this class of medication ( $n=129)$ showed no statistically significant differences between the groups. It has been found that GLP-1 agonists can therefore be continually used according to the current standard protocols for colonoscopy before and after colonoscopy, but no conclusions were made regarding the impact of the procedure on the occurrence of hypoglycaemia [30].

Frequent blood glucose monitoring on the day preceding the examination and on the day of the examination should be an important element of the colonoscopy preparation process. It is recommended that patients treated with insulin should monitor their glucose levels at least every $4 \mathrm{~h}$ from the last solid meal until eating again after the procedure [11]. Some more restrictive recommendations indicate the need to monitor glucose levels even every hour in the periprocedural period [31].

Currently, however, the main challenge for patients with diabetes and other metabolic disorders preparing for endoscopy is adherence to the examination preparation recommendations. The use of a liquid diet ensuring adequate carbohydrate intake and the appropriate adjustment of the dosage of antidiabetic drugs enables the maintenance of glycaemic control, which is important for the condition of patients and their compliance with the recommendations regarding preparation for the examination. It is also important 
to control blood glucose levels, especially in patients taking insulin. Considering the lack of guidelines on preparation for examinations of the lower gastrointestinal tract in the discussed group of patients, it is necessary to carry out in-depth research on dietary modifications, changes in the endoscopy hours, the use of additional preparations or modifications to the existing ones, which may ensure the proper quality of bowel cleansing. It should also be emphasized that appropriate patient education is essential for their adherence to the recommendations regarding the endoscopy preparatory procedures.

\section{Summary}

The current guidelines for preparatory procedures for endoscopic examinations mainly apply to the general population, and there are no specific recommendations for patients with metabolic disorders, especially those with diabetes and at high risk of hypoglycaemia. There are suggestions in some scientific studies, but they are not sufficiently systematized. The recommendations dedicated to patients with diabetes mainly include the appropriate use of antidiabetic drugs, which, considering that the preparation of patients for endoscopic examinations is conducted at home, may contribute to certain complications.

Diabetes mellitus, as one of the metabolic diseases, has been shown to be an important factor that increases the likelihood of improper bowel preparation. There are several studies confirming a significantly higher percentage of improperly prepared patients in the diabetic population compared to the reference (healthy) population. The causes of this phenomenon can be found in the pathophysiology of diabetes, characterized by slow intestinal transit, and thus difficult intestinal cleansing process, as well as difficulties in adhering to dietary recommendations in the period of preparation for colonoscopy, because they are not adapted to the special needs of people at risk of developing episodes of hypoglycaemia.

Incorrect bowel preparation for colonoscopy may require the procedure to be repeated, which incurs additional costs on the health care system, extends the waiting time for a colonoscopy, and is associated with reduction of the patient's quality of life, because it requires re-undergoing the burdensome procedure of preparing for an invasive examination. An examination carried out on an improperly cleaned bowel increases the risk of overlooking existing neoplastic lesions, intestinal polyps, or minor mucosa damage that could lead to the development of cancer. Inadequate bowel cleansing also significantly extends the duration of the examination and is associated with increased patient discomfort, as well as an increased likelihood of adverse events associated with the procedure.

Moreover, a very important aspect that influences patients' motivation to perform endoscopic examinations is the stress and anxiety associated with both the examination itself and, in particular, the process of intestinal preparation. Research shows that these negative feelings become of moderate to severe intensity, often leading to avoidance or delay of the endoscopic examination, which may result in a late diagnosis and loss of a chance for an effective treatment. There are articles describing methods of adapting the standard protocol of preparation for endoscopy to the needs of diabetic patients by changing the regimen of use, or using additional laxatives, modifying dietary recommendations, altering dosing of antidiabetic agents, or modifying the time of the examination. They are a step on the way to creating the procedure that takes into account the needs of people with metabolic diseases, enabling effective and safe preparation for endoscopic examination.

The publications cited in the review mostly refer to diabetic patients undergoing colonoscopy. It should be borne in mind that the conclusions of the review may also apply to the general population of people with metabolic disorders preparing for surgeries and other procedures requiring optimal bowel preparation, similar to colonoscopy.

Considering the constantly growing number of the above-described group as well as the fact that some metabolic diseases (especially the most common diabetes) predispose to an increased risk of developing colorectal cancer, it can be said that the lack of precise recommendations regarding preparation for endoscopic procedures tailored to the specific needs of the discussed population constitute a significant gap in the health care system. It even means that the recommendations regarding the procedure to be followed given by individual doctors may be contradictory. It also leads to the fact that another important element of preparing the bowel for examination, i.e. proper patient education and information, cannot be properly implemented. At the same time, it is estimated that inadequate bowel cleansing may increase the hospitalization time of inpatients by $25 \%$, and the costs of these hospitalizations by $30 \%$ [32].

The introduction of a management regimen adapted for patients with metabolic disorders into clinical practice may allow the exclusion (or minimization of the impact) of factors that may potentially be the cause of poor preparation for endoscopic examinations, and they may contribute to the identification of other factors negatively affecting the preparation of the intestine for endoscopy. 
This publication was created as part of the project entitled "Product for patients with metabolic disorders preparing for endoscopic examinations", implemented by BioGliko Sp. z o.o. as part of the Smart Growth Operational Programme, Support Agreement No. 2/2019 of 23.07.019.

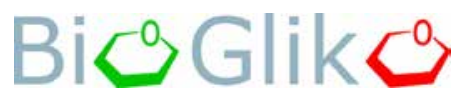

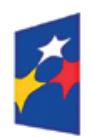
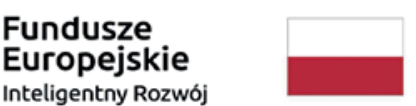

Rzeczpospolita Polska

\section{References}

1. Hassan C, East J, Radaelli F, et al. Bowel preparation for colonoscopy: European Society of Gastrointestinal Endoscopy (ESGE) Guideline - Update 2019. Endoscopy 2019; 51: 775 94.

2. Murphy D, Jenks M, McCool R, et al. A systematic review and cost analysis of repeat colonoscopies due to inadequate bowel cleansing in five European countries. Expert Rev Pharmacoeconom Outcomes Res 2019; 19: 701-9.

3. Lee B, Jain D, Rajala M. A survey of peri-colonoscopy management of anti-diabetic medications. Clin Endosc 2020; 53: 623-6.

4. Kamiński MF, Hassan C, Bisschops R, et al. Advanced imaging for detection and differentiation of colorectal neoplasia: European Society of Gastrointestinal Endoscopy (ESGE) Guideline. Endoscopy 2014; 46: 435-49.

5. Bechtold ML, Mir F, Puli SR, et al. Optimizing bowel preparation for colonoscopy: a guide to enhance quality of visualization. Ann Gastroenterol 2016; 29: 137-46.

6. Zad M, Do C, Heffernan A, et al. Factors affecting bowel preparation adequacy and procedural time. JGH Open 2019; 4: 20614.

7. Shah P, Alsayed A, Ahmed W, et al. Impact of colonoscopy in patients older than 75 years. J Geriatr Med Gerontol 2020; 6: 93.

8. Łaski, D, Perdyan A, Spychalski P, et al. Patients seeking information about colonoscopy - lessons learned from Google. Gastroenterology Rev 2020; 15: 144-50.

9. Pilonis ND, Bugajski M, Wieszczy P, et al. Long-term colorectal cancer incidence and mortality after a single negative screening colonoscopy. Ann Intern Med 2020; 173: 81-91.

10. Franczyk R. Przygotowanie do kolonoskopii wybranych grup pacjentów. Gastroenterol Klin Postępy Stand 2018; 10: 151-5.

11. Hochberg I, Segol O, Shental R, et al. Antihyperglycemic therapy during colonoscopy preparation: a review and suggestions for practical recommendations. United European Gastroenterol J 2019; 7: 735-40.

12. Alvarez-Gonzalez M, Flores-Le Roux J, Seoane A, et al. Efficacy of a multifactorial strategy for bowel preparation in diabetic patients undergoing colonoscopy: a randomized trial. Endoscopy 2016; 48: 1003-9.

13. Zdrojewski Ł, Zdrojewski T, Rutkowski M, et al. Prevalence of chronic kidney disease in a representative sample of the Polish population: results of the NATPOL 2011 survey. Nephrol Dial Transplant 2016; 31: 433-9.
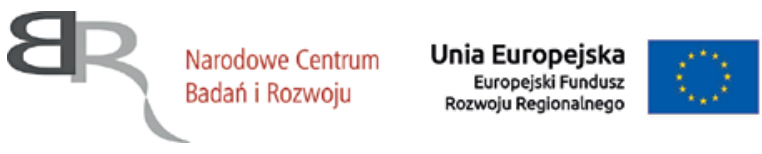

14. Kalbarczyk WP. Cukrzyca gdzie jesteśmy? Dokąd zmierzamy? Raport Instytutu Ochrony Zdrowia. 2018

15. International Diabetes Federation (IDF) (2017) IDF Diabetes Atlas. 8th Edition, International Diabetes Federation, Brussels. http://www.diabetesatlas.org/resources/2017-atlas.html

16. Sørensen M, Johansen OE. Idiopathic reactive hypoglycaemia - prevalence and effect of fibre on glucose excursions. Scand J Clin Lab Invest 2010; 70: 385-91.

17. Simpson EJ, Holdsworth M, Macdonald IA. Prevalence of selfreported symptoms attributed to hypoglycaemia within a general female population of the UK. J Psychosom Res 2006; 60: 403-6.

18. Nirantharakumar K, Marshall T, Hodson J, et al. Hypoglycemia in non-diabetic in-patients: clinical or criminal? PLoS One 2012; 7: e40384.

19. Sako A, Yasunaga H, Matsui H, et al. Hospitalization with hypoglycemia in patients without diabetes mellitus: a retrospective study using a national inpatient database in Japan, 2008-2012. Medicine 2017; 96: e7271.

20. Jakubowski A, Kamiński M, Müllner K, et al. Stosowanie analgosedacji do endoskopii w Polsce. Postęp Nauk Med 2009; 2: 88-98.

21. Larsson SC, Orsini N, Wolk A. Diabetes mellitus and risk of colorectal cancer: a meta-analysis. J Natl Cancer Inst 2005; 97: 1679-87.

22. Taylor C, Schubert ML. Decreased efficacy of polyethylene glycol lavage solution (golytely) in the preparation of diabetic patients for outpatient colonoscopy: a prospective and blinded study. Am J Gastroenterol 2001; 96: 710-4.

23. Ozturk NA, Gokturk HS, Demir M, et al. The effect of autonomous neuropathy on bowel preparation in type 2 diabetes mellitus. Int J Colorectal Dis 2009; 24: 1407-12.

24. Hilsden RJ, Bridges R, Dube C, et al. Scheduling rules for patients with diabetes mellitus that facilitate split-dosing improve the quality of bowel preparation for colonoscopy. PLoS One 2017; 12: e0182225.

25. Kim Y, Seo E, Lee J, et al. Inadequate bowel cleansing efficacy of split-dose polyethylene glycol for colonoscopy in type 2 diabetic patients: a prospective and blinded study. J Clin Gastroenterol 2017; 51: 240-6.

26. Madhoun MF, Bitar H, Bhatti O, et al. Diabetics on narcotics are less likely to achieve excellent bowel preparation than are patients with either condition. Dig Dis Sci 2017; 62: 723-9.

27. Mahmood S, Farooqui S, Madhoun M. Predictors of inadequate bowel preparation for colonoscopy: a systematic re- 
view and meta-analysis. Eur J Gastroenterol Hepatol 2018; 30: 819-26.

28. Hayes A, Buffum M, Hughes J. Diabetic colon preparation comparison study. Gastroenterol Nurs 2011; 34: 377-82.

29. Grigg E, Schubert M, Hall J, et al. Lubiprostone used with polyethylene glycol in diabetic patients enhances colonoscopy preparation quality. World J Gastrointest Endosc 2010; 2: 263-7.

30. Sharma T, Das N, Ismail B, et al. Evaluation of the effect of GLP-1 agonists on quality of bowel preparation for colonoscopy in patients with diabetes. Pract Diabetes 2017; 34: 167-8.

31. Australian Diabetes Society. Australian Diabetes Society Guidelines for Routine Glucose Control in Hospital. Australian Diabetes Society 2012.

32. Fuccio L, Frazzoni L, Spada C, et al. Factors that affect adequacy of colon cleansing for colonoscopy in hospitalized patients. Clin Gastroenterol Hepatol 2021; 19: 339-48.

Received: 15.11 .2021

Accepted: 30.11 .2021 